\title{
Intravitreal injection of conbercept combined with retinal photocoagulation on macular edema secondary to branch retinal vein occlusion
}

\author{
BING WANG $^{1 *}$, SHUFEN FANG $^{2 *}$ and LING CHEN ${ }^{3}$ \\ ${ }^{1}$ Department of Ophthalmology, Yantaishan Hospital, Yantai, Shandong 264000; \\ ${ }^{2}$ Department of Ophthalmology, Laizhou People's Hospital of Yantai, \\ Yantai, Shandong 261400; ${ }^{3}$ Department of Ophthalmology, \\ Affiliated to Qingdao University Yuhuangding Hospital of Yantai, \\ Yantai, Shandong 264000, P.R. China
}

Received October 24, 2019; Accepted February 12, 2020

DOI: $10.3892 /$ etm.2020.8660

\begin{abstract}
Effects of Conbercept combined with retinal photocoagulation on macular edema secondary to branch retinal vein occlusion (BRVO) were investigated. A total of 98 patients (98 eyes) with macular edema secondary to BRVO were collected. The central macular thickness (CMT), incidence rate of complications after treatment and best corrected visual acuity (BCVA) were recorded. Also the factors affecting visual recovery of patients were analyzed. At 1 week, 1 month, 3 months and 6 months after treatment, the BCVA in both groups was significantly superior to that before treatment $(\mathrm{P}<0.05)$. In the combination group and laser group, the logarithm of the minimum angle of resolution (logMAR) of BCVA increased from $0.84 \pm 0.47$ to $0.34 \pm 0.10$ and from $0.89 \pm 0.49$ to $0.45 \pm 0.14$, and CMT declined from $559.5 \pm 152.7$ to $267.8 \pm 19.8 \mu \mathrm{m}$ and from $570.3 \pm 172.6$ to $314.7 \pm 18.4 \mu \mathrm{m}$. It was observed that at 1 week, 1 month, 3 months and 6 months after treatment, the BCVA in combination group was obviously better than that in laser group $(\mathrm{P}=0.008, \mathrm{P}<0.001, \mathrm{P}=0.004, \mathrm{P}<0.001$, respectively), while CMT in combination group was obviously smaller than that in laser group $(\mathrm{P}=0.009, \mathrm{P}=0.002, \mathrm{P}<0.001, \mathrm{P}<0.001)$. Conbercept with retinal photocoagulation can effectively improve the visual acuity and reduce the CMT. The visual recovery of patients after treatment is related to the BCVA before treatment, decreased value of CMT at 1 month after treatment and integrity of external limiting membrane (ELM).
\end{abstract}

Correspondence to: Dr Ling Chen, Department of Ophthalmology, Affiliated to Qingdao University Yuhuangding Hospital of Yantai, 20 YuHuangDing East Road, Yantai, Shandong 264000, P.R. China E-mail: wohod119@163.com

${ }^{*}$ Contributed equally

Key words: branch retinal vein occlusion, macular edema, Conbercept, laser photocoagulation, efficacy

\section{Introduction}

Branch retinal vein occlusion (BRVO) is a common retinal vascular disease, and the secondary macular edema (ME) is an important cause of visual impairment $(1,2)$. The treatment of BRVO aims to alleviate ME, inhibit angiogenesis, and eliminate non-perfusion area, in which early alleviation of $\mathrm{ME}$ is the key to improving visual acuity $(3,4)$. At present, the most commonly used therapeutic methods for ME include intravitreal injection of triamcinolone acetonide, intravitreal injection of anti-vascular endothelial growth factor (VEGF) drugs, and fundus laser photocoagulation (5). Macular grid laser photocoagulation can reduce vascular leakage and alleviate ME, but its improvement effect on visual acuity is limited, with a risk of visual impairment (6). Intravitreal injection of anti-VEGF drugs can effectively treat RVO, and combined with laser photocoagulation can achieve better results $(7,8)$.

This study retrospectively analyzed the clinical data of 98 patients with BRVO secondary macular edema admitted to Yantaishan Hospital (Yantai, China) from June 2017 to June 2018. Comparative analysis of retinal photocoagulation and intravitreal injection of Conbercept combined with retinal photocoagulation was carried out. Therapeutic efficacy and safety of BRVO secondary macular edema was evaluated in order to provide a more accurate basis for the development of effective treatment options.

\section{Patients and methods}

General data. A total of 98 patients (98 eyes) diagnosed with ME secondary to BRVO in Yantaishan Hospital from June 2017 to June 2018 were selected, including 51 males and 47 females

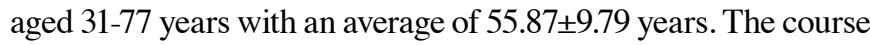
of disease was 5 days to 3 months, and the best corrected visual acuity (BCVA) before treatment and central macular thickness (CMT) were 0.4 and 351.4-731.8 $\mu \mathrm{m}$ with an average of $563.6 \pm 162.5 \mu \mathrm{m}$, respectively. The patients were divided into laser group (retinal photocoagulation alone, $\mathrm{n}=49$ ) and combination group (intravitreal injection of Conbercept combined with retinal 
photocoagulation, $n=49$ ) using a random number table based on the treatment order. Inclusion criteria: i) fluorescein fundus angiography (FFA) was diagnosed as BRVO; ii) optical coherence tomography (OCT) showed macular cystic edema, and the retina was diffusely thickened; iii) All are monocular, with a course of $<3$ months. Exclusion criteria: i) previous exposure to intravitreal injection of anti-VEGF drugs or subconjunctival injection of triamcinolone acetonide; ii) previous treatment with laser photocoagulation; iii) diagnosis or suspected shallow anterior chamber, glaucoma or ocular hypertension; iv) ME secondary to age-related macular degeneration, diabetic retinopathy, retinal vein inflammation, retinal vasculitis; v) eye diseases other than BRVO; vi) history of drug allergy and bronchi asthma, and diabetes. The general data, such as sex, age, BCVA before treatment, CMT and intraocular pressure, had no statistically significant differences between the two groups $(\mathrm{P}>0.05$, Table I), and they were comparable. This study was approved by the Ethics Committee of Yantaishan Hospital, and patients and their families agreed and signed the informed consent.

Treatment methods. In combination group, intravitreal injection of Conbercept was performed routinely. $0.05 \mathrm{ml}$ of Conbercept injection $(10.0 \mathrm{mg} / \mathrm{ml})$ containing $0.5 \mathrm{mg}$ of Conbercept was taken using a $1 \mathrm{ml}$ syringe, and the syringe was vertically inserted at $3.75 \mathrm{~mm}$ behind the corneal limbus to slowly inject Conbercept. After that, Ofloxacin eye ointment was applied and the eye was bandaged. Whether the treatment should be repeated after injection once was determined according to the return visit examination. The anti-VEGF drugs were given if the BCVA declined by 2 lines or CMT was increased by $\geq 100 \mu \mathrm{m}$. The injection was performed twice at an interval of $\geq 4$ weeks. At 1 week after injection, the patients underwent laser photocoagulation under a retinoscope using the multi-wavelength laser machine (Lumenis). The laser parameters are as follows: spot diameter, 100-200 $\mu \mathrm{m}$; power, 150-200 mW; exposure time, 0.1-0.15 sec; level I spot, spot gap, $\sim 1$ spot diameter. The quadrant photocoagulation was conducted for the non-perfusion area shown in FFA. The laser parameters are as follows: spot diameter, 50-100 $\mu \mathrm{m}$; exposure time, $0.2 \mathrm{sec}$; spot reaction, level I-II, spot gap, $\sim 1$ spot diameter. After treatment, eye drops (non-steroidal anti-inflammatory drugs) were used 4 times per day for 3 consecutive days. The range of photocoagulation each time was $\leq 1 / 4$ quadrant, and photocoagulation was conducted twice at an interval of 1 week. The patients who could not receive photocoagulation due to great bleeding should undergo photocoagulation after bleeding absorption.

In laser group, the macular grid laser photocoagulation was directly performed. The laser parameters are as follows: spot diameter, 100-200 $\mu \mathrm{m}$; power, 150-200 mW; exposure time, 0.1-0.15 sec; level I spot, spot gap: 1 spot diameter. Quadrant local photocoagulation was performed using the same laser parameters for the patients with non-perfusion area or new vessels in the peripheral retina. All operations were completed by the same physician.

Observation indexes. The BCVA, OCT, fundus color photography, and FFA examination were performed before treatment and 1 week and 1, 3, and 6 months after treatment. The BCVA examination uses a standard logarithmic visual acuity chart, which is converted to the logarithm of the minimum angle of resolution $(\log$ MAR) visual acuity. Nonmyd $\alpha$-DIII non-mydriatic fundus camera (Kowa Co., Ltd.) was used in fundus color photography, Spectralis HRA instrument was used in FFA, and Spectralis OCT instrument was used in OCT. With the central fovea of macula as the center, the horizontal linear scanning was conducted (scanning depth, $1.9 \mathrm{~mm}$; scanning area, 6x6 mm; transverse resolution, $14 \mu \mathrm{m}$; axial resolution, $7 \mu \mathrm{m}$; scanning mode, 512x496). The distance from the internal limiting membrane of retinal nerve epithelial layer to the lateral strong reflective area of retinal pigment epithelial layer was measured as CMT using the built-in range of software by two experienced technicians 3 times, and the average was taken. The integrity of ellipsoid zone and external limiting membrane (ELM) was observed on the central horizontal scanning line and within $500 \mu \mathrm{m}$ in the greyscale map. We also recorded the BCVA, mean CMT changes, intraocular pressure changes, and complications before and 1 week after treatment and at 1,3, and 6 months.

The correlations of long-term visual acuity of patients in combination group with age, logMAR BCVA before treatment, CMT before treatment, times of drug injection and decrease value of CMT at 1 month after treatment were analyzed using Pearson correlation analysis. The multivariate regression analysis was adopted for the correlations of long-term visual acuity of patients in combination group with age, $\log$ MAR BCVA before treatment, CMT before treatment, times of drug injection, decreased value of CMT at 1 month after treatment, and integrity of ellipsoid zone and ELM.

Statistical analysis. Statistical Product and Service Solutions (SPSS) 22.0 software (IBM Corp.) was used for statistical analysis. Enumeration data were expressed as rate $(\%)$, and $\chi^{2}$ test was performed for the intergroup comparison. Measurement data were expressed as mean \pm standard deviation (mean \pm SD), multivariate analysis of variance (MANOVA) with repeated measures was adopted for the comparison of BCVA and CMT at different time points, independent-samples t-test for the intergroup comparison, and LSD-t test for the intragroup comparison. $\mathrm{P}<0.05$ was considered to indicate a statistically significant difference.

\section{Results}

Changes in BCVA before and after treatment in the groups. The changes in BCVA had statistically significant differences in both groups before and after treatment ( $\mathrm{F}$ group $=14.56$, $\mathrm{F}$ time $=10.84, \mathrm{~F}$ time $\mathrm{x}$ group $=12.62, \mathrm{P}<0.01)$. At 1 week, 1 month, 3 months and 6 months after treatment, the BCVA in both groups was significantly superior to that before treatment $(\mathrm{P}<0.05)$, and the BCVA in combination group was obviously better than that in laser group, showing statistically significant differences $(\mathrm{P}=0.008, \mathrm{P}<0.001, \mathrm{P}=0.004, \mathrm{P}<0.001)$ (Fig. 1).

Changes in CMT before and after treatment in the groups. The changes in CMT had statistically significant differences in both groups before and after treatment ( $\mathrm{F}$ group $=13.47$, F time $=17.28$, F time $x$ group $=20.34, \mathrm{P}<0.01)$, and $\mathrm{ME}$ obviously subsided after treatment (Fig. 2). At 1 week, 1, 3 and 6 months after treatment, CMT evidently declined in both 
Table I. Demographics and general clinical data of the studied patients.

\begin{tabular}{lccc}
\hline Parameters & $\begin{array}{c}\text { Combination group } \\
\mathrm{n}=49\end{array}$ & $\begin{array}{c}\text { Laser group } \\
\mathrm{n}=49\end{array}$ \\
\hline Sex (male/female) & $27 / 22$ & $24 / 25$ & 0.686 \\
Age (years) & $55.13 \pm 9.62$ & $56.84 \pm 9.57$ & 0.380 \\
Course of the disease (days) & $19.5 \pm 2.7$ & $18.8 \pm 2.3$ & 0.170 \\
BCVA (logMAR) & $0.84 \pm 0.47$ & $0.89 \pm 0.49$ & 0.607 \\
CMT $(\mu$ m) & $559.5 \pm 152.7$ & $570.3 \pm 172.6$ & 0.744 \\
IOP (mmHg) & $14.8 \pm 3.9$ & $15.2 \pm 3.5$ & 0.594 \\
Occlusion vessel & & $26(53.1 \%)$ \\
Superior temporal branch & $23(46.9 \%)$ & $14(28.6 \%)$ \\
Inferior temporal branch & $17(34.7 \%)$ & $3(6.1 \%)$ \\
Superior nasal branch & $4(8.2 \%)$ & $6(12.2 \%)$ \\
Inferior nasal branch & $5(10.2 \%)$ & 0.871 \\
\hline
\end{tabular}

BCVA, best corrected visual acuity; logMAR, logarithm of the minimum angle of resolution; CMT, central macular thickness; IOP, intraocular pressure.

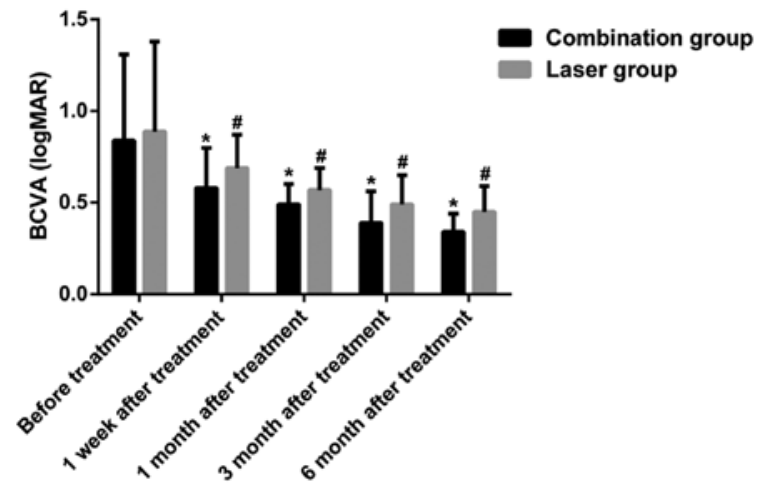

Figure 1. Comparison of pretreatment and post-treatment BCVA (logMAR) of the studied patients in two different groups. "Compared with laser group, $\mathrm{P}<0.05$; "compared with before treatment, $\mathrm{P}<0.05$. BCVA, best corrected visual acuity; logMAR, logarithm of the minimum angle of resolution.

groups compared with that before treatment $(\mathrm{P}<0.05)$, while it was obviously smaller in combination group than that in laser group $(\mathrm{P}=0.009, \mathrm{P}=0.002, \mathrm{P}<0.001, \mathrm{P}<0.001)$ (Fig. 3).

Changes in intraocular pressure before and after treatment in the groups. No statistically significant difference was found in intraocular pressure between and within the two groups before and after treatment $(\mathrm{P}>0.05)$ (Fig. 4).

Times of drug injection and related complications in the groups. In combination group, the average times of intravitreal injection in affected eyes were $2.43 \pm 0.86$ times, and 31 eyes received intravitreal injection 2-4 times. During treatment and follow-up, no ocular complications (intraocular hypertension, endophthalmitis, vitreous hemorrhage, retinal tear and iatrogenic cataract) and systemic adverse reactions occurred.

Influencing factors for visual recovery in combination group after treatment. According to the Pearson correlation analysis, the visual recovery of patients in combination group was
Table II. Pearson correlation analysis and multiple regression analysis of the studied patients in combination treatment group.

\begin{tabular}{lcc}
\hline Factors & r-value & P-value \\
\hline Pearson correlation analysis & & \\
Age & 0.087 & 0.695 \\
Pretreatment logMAR BCVA & 0.434 & 0.001 \\
Pretreatment CMT $(\mu \mathrm{m})$ & -0.172 & 0.506 \\
Injection times & 0.348 & 0.001 \\
One month posttreatment CMT $(\mu \mathrm{m})$ & 0.593 & 0.001 \\
Multiple regression analysis & & \\
Age & -0.310 & 0.784 \\
Pretreatment logMAR BCVA & 5.525 & 0.001 \\
Pretreatment CMT $(\mu \mathrm{m})$ & 2.103 & 0.074 \\
Injection times & 0.418 & 0.570 \\
1 month posttreatment CMT $(\mu \mathrm{m})$ & 6.763 & 0.001 \\
ELM completeness & -0.425 & 0.001 \\
EZ completeness & -1.790 & 0.181 \\
\hline
\end{tabular}

$\log$ MAR, logarithm of the minimum angle of resolution; BCVA, best corrected visual acuity; CMT, central macular thickness; ELM, external limiting membrane; EZ, ellipsoid zone.

positively correlated with the logMAR BCVA before treatment, decrease value of CMT at 1 month after treatment and times of drug injection $(\mathrm{P}<0.05)$, while it was not correlated with the age or CMT before treatment $(\mathrm{P}>0.05)$. The results of multivariate regression analysis revealed that the visual recovery of patients in combination group was associated with the logMAR BCVA before treatment, decreased value of CMT at 1 month after treatment, integrity of ELM and times of drug injection $(\mathrm{P}<0.05)$, while it was not associated with the age, CMT before treatment or integrity of ellipsoid zone ( $\mathrm{P}>0.05)$ (Table II). 

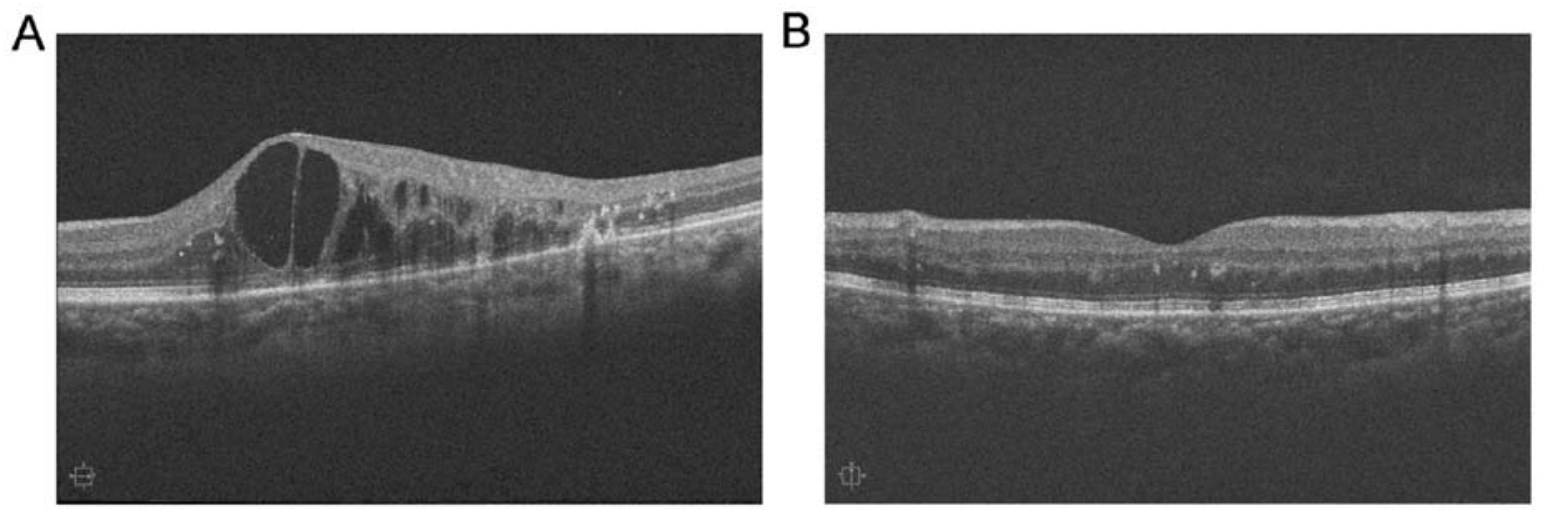

Figure 2. Comparison of pretreatment and 6 months post-treatment OCT of a macular edema secondary to BRVO patient. (A) Pretreatment OCT of the patient indicates severe neuro-epithelium detachment and macular cystoid edema. (B) OCT of the patient 6 months after intravitreal Conbercept injection combined with retinal laser photocoagulation showed macular edema dramatically subsided and retinal structure almost recovered to normal. OCT, optical coherence tomography; BRVO; branch retinal vein occlusion.

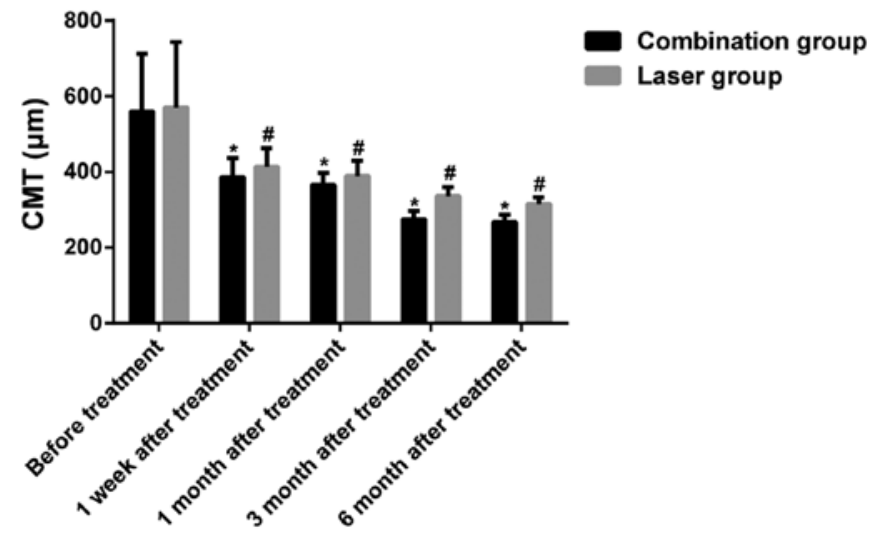

Figure 3. Comparison of pretreatment and post-treatment CMT $(\mu \mathrm{m})$ of the studied patients in two different groups. ${ }^{*}$ Compared with laser group, $\mathrm{P}<0.05$; ${ }^{\#}$ Compared with before treatment, $\mathrm{P}<0.05$. CMT, central macular thickness.

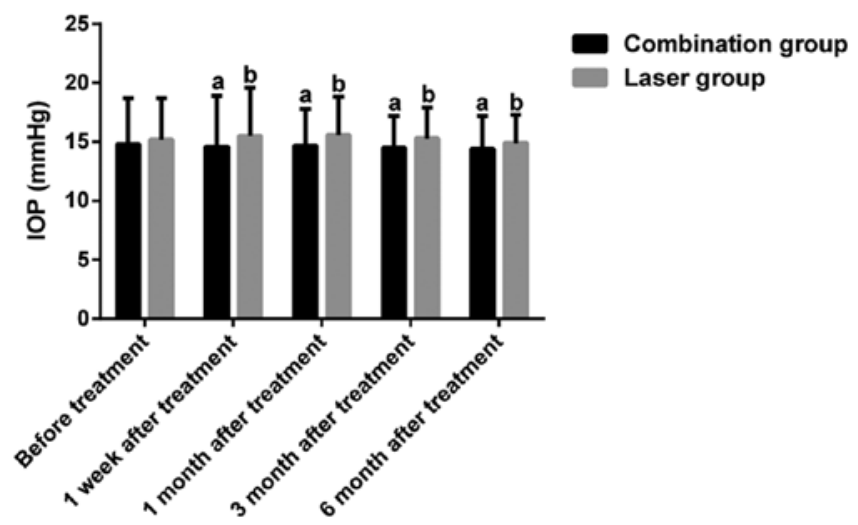

Figure 4. Comparison of pretreatment and post-treatment IOP $(\mathrm{mmHg})$ of the studied patients in the two groups. ${ }^{a}$ Compared with laser group, $\mathrm{P}>0.05$; ${ }^{\mathrm{b}}$ Compared with before treatment, $\mathrm{P}>0.05$. IOP, intraocular pressure.

\section{Discussion}

Secondary ME is the major cause of diminution of vision in BRVO patients, and its pathogenesis is mainly retinal circulatory disorder and venous circuity and distention, in which the local ischemia and hypoxia lead to the destruction of the retinal blood-oxygen barrier and the damage of retinal pigment epithelial cells, and the fundus microangiopathy results in leakage, thus causing retinal tissue edema and $\mathrm{ME}$ when involving macula (9). It has been found in recent studies that the level of VEGF in the vitreous body of BRVO patients is higher, and the high expression of VEGF leads to exudation of serum protein, and promotes angiogenesis and ME. Therefore, anti-VEGF can alleviate and treat ME secondary to BRVO $(10,11)$. Conbercept, a kind of fully humanized recombinant fusion protein, can block all VEGF-A subtypes, VEGF-B and placental growth factor, reduce vascular leakage, and inhibit vascular endothelial cell proliferation and angiogenesis, thereby treating ME (12). Multiple studies have shown that intravitreal injection of Conbercept can rapidly improve the visual acuity of patients with ME secondary to RVO, but the duration of action of this drug is limited, and multiple injections are required, which causes a certain economic burden for patients (13-15).

Laser photocoagulation is a conventional treatment means for BRVO, its mechanism is that due to the destruction of photoreceptor cells and retinal pigment epithelial cells, the oxygen content in the inner retina relatively increases, the contraction of arterioles is caused, and the pressure of capillary network and vein relatively declines, thereby reducing vascular leakage and alleviating ME (16). However, it is difficult to perform laser photocoagulation in the case of severe $\mathrm{ME}$ and retinal edema, and great bleeding, and it has been confirmed that the affected eyes often suffer from visual field defect and decline in contrast sensitivity after laser photocoagulation, affecting the recovery of visual function (17). Therefore, some scholars have proposed that combining laser photocoagulation and anti-VEGF drugs can not only improve the efficacy on ME, but also facilitate the recovery of visual function of patients, which has high clinical application value.

Our study compared the efficacy and safety of simple retinal photocoagulation with intravitreal injection of Conbercept combined with retinal photocoagulation for the treatment of macular edema secondary to BRVO. It was found that CMT was significantly smaller in combination group than that in laser group at each time point after treatment, and the possible reason is that Conbercept inhibits pathological 
neovascularization, induces differentiation, increase migration of vascular endothelial cells, improves vascular endothelial cell function, reduces the elimination of retinal endothelial system on granular antigens, and repairs the structure and function of blood-retinal barrier, thereby alleviating fluid exudation and inflammatory response (18). Moreover, the scar tissues produced by retinal photocoagulation maintain the blood-retinal barrier, promote the absorption of diffuse ME, and reduce the lesions, thus decreasing CMT (19). In addition, the BCVA was also remarkably improved in combination group compared with that in laser group at each time point after treatment, and the possible reason is that Conbercept was used to alleviate ME before retinal photocoagulation, providing an optimal opportunity for photocoagulation. In the case of RVO secondary to macular edema, the Conbercept is given via intravitreal injection. Because of the rapid onset and strong effect of anti-VEGF treatment, it can quickly and significantly reduce macular edema, and can make the cornea-like photocoagulation in the macular area. Low energy is more efficiently accomplished, achieving better therapeutic results with smaller lesions, thereby reducing the frequency of treatment, avoiding recurrence and improving vision in patients with RVO macular edema (20).

In this study, the results showed that the visual recovery was not associated with age and CMT before treatment, but positively correlated with BCVA before treatment. A study has proved that the course of disease is associated with the degree of visual recovery, so only those patients with a course $<3$ months were enrolled in this study (21). Besides, research has revealed that the simple fracture of ellipsoid zone can repair autonomously, but beyond recovery if it is combined with ELM damage, suggesting that ELM plays an important role in the damage repair of ellipsoid zone (22). In this study, the results manifested that the integrity of ellipsoid zone had no correlation with visual recovery after treatment. However, Inoue et al (23) found that there are still repair of ellipsoid zone and visual recovery at 12 months after treatment. The reason may be related to the short observation time in this study. Moreover, it was observed that the integrity of ELM may play a significant role in the recovery of visual function, and the changes in CMT and the structural integrity of ELM at 1 month after the first treatment are of certain value in the prognosis evaluation of visual acuity.

However, the results in this study need to be further verified using large numer of samples in multi-center randomized controlled trials.

In conclusion, intravitreal injection of Conbercept combined with retinal photocoagulation can effectively improve the visual acuity and reduce the CMT of patients. The visual recovery of patients after treatment is correlated with the BCVA before treatment, decreased value of CMT at 1 month after treatment and integrity of ELM.

\section{Acknowledgements}

Not applicable.

\section{Funding}

Not applicable.

\section{Availability of data and materials}

All data generated or analyzed during this study are included in this published article.

\section{Authors' contributions}

BW and LC designed the study and performed the experiments, SF and LC collected the data, BW and SF analyzed the data, BW and LC prepared the manuscript. All authors read and approved the final manuscript.

\section{Ethics approval and consent to participate}

This study was approved by the Ethics Committee of Yantaishan Hospital (Yantai, China). Signed informed consents were obtained from all participants before the study.

\section{Patient consent for publication}

Patients or their guardians provided written informed consent for publication.

\section{Competing interests}

The authors declare they have no competing interests.

\section{References}

1. Yau JW, Lee P, Wong TY, Best J and Jenkins A: Retinal vein occlusion: An approach to diagnosis, systemic risk factors and management. Intern Med J 38: 904-910, 2008.

2. Pierru A, Girmens JF, Héron E and Paques M: Retinal vein occlusions. J Fr Ophtalmol 40: 696-705, 2017 (In French).

3. Jaulim A, Ahmed B, Khanam T and Chatziralli IP: Branch retinal vein occlusion: Epidemiology, pathogenesis, risk factors, clinical features, diagnosis, and complications. An update of the literature. Retina 33: 901-910, 2013.

4. Feltgen $\mathrm{N}$ and Pielen A: [Retinal vein occlusion: Epidemiology, classification and clinical findings]. Ophthalmologe 112: 607-618, quiz 619-620, 2015 (In German).

5. Ehlers JP, Kim SJ, Yeh S, Thorne JE, Mruthyunjaya P, Schoenberger SD and Bakri SJ: Therapies for macular edema associated with branch retinal vein occlusion: A report by the American Academy of Ophthalmology. Ophthalmology 124: 1412-1423, 2017.

6. Spaide RF: Retinal vascular cystoid macular edema: Review and new theory. Retina 36: 1823-1842, 2016.

7. Campochiaro PA, Heier JS, Feiner L, Gray S, Saroj N, Rundle AC, Murahashi WY and Rubio RG; BRAVO Investigators: ranibizumab for macular edema following branch retinal vein occlusion: Six-month primary end point results of a phase III study. Ophthalmology 117: 1102-1112.e1, 2010.

8. Tadayoni R, Waldstein SM, Boscia F, Gerding H, Gekkieva M, Barnes E, Das Gupta A, Wenzel A and Pearce I; BRIGHTER Study Group: Sustained benefits of ranibizumab with or without laser in branch retinal vein occlusion: 24-month results of the BRIGHTER Study. Ophthalmology 124: 1778-1787, 2017.

9. Daruich A, Matet A, Moulin A, Kowalczuk L, Nicolas M, Sellam A, Rothschild PR, Omri S, Gélizé E, Jonet L, et al: Mechanisms of macular edema: Beyond the surface. Prog Retin Eye Res 63: 20-68, 2018.

10. Chen $\mathrm{CH}$, Chen YH, Wu PC, Chen YJ, Lee JJ, Liu YC and Kuo HK: Treatment of branch retinal vein occlusion induced macular edema in treatment-naïve cases with a single intravitreal triamcinolone or bevacizumab injection. Chang Gung Med J 33: 424-435, 2010.

11. Feng J, Zhao T, Zhang Y, Ma Y and Jiang Y: Differences in aqueous concentrations of cytokines in macular edema secondary to branch and central retinal vein occlusion. PLoS One 8: e68149, 2013. 
12. Xia JP, Wang S and Zhang JS: The anti-inflammatory and anti-oxidative effects of conbercept in treatment of macular edema secondary to retinal vein occlusion. Biochem Biophys Res Commun 508: 1264-1270, 2019.

13. Sun Z, Zhou H, Lin B, Jiao X, Luo Y, Zhang F, Tao S, Wu Q, $\mathrm{Ke} Z$ and Liu X: Efficacy and safety of intravitreal Conbercept injections in macular edema secondary to retinal vein occlusion. Retina 37: 1723-1730, 2017.

14. Tang F, Qin X, Lu J, Song P, Li M and Ma X: Optical coherence tomography predictors of short-term visual acuity in eyes with macular edema secondary to retinal vein occlusion treated with intravitreal conbercept. Retina: Jan 10, 2019 (Epub ahead of print).

15. Luo W, Jia F, Liu M, Wang Y and Zhang T: The analysis of correlative factors of visual acuity with intravitreal Conbercept injection in macular edema associated with branch retinal vein occlusion. J Ophthalmol 2018: 7348153, 2018.

16. Tadayoni R, Waldstein SM, Boscia F, Gerding H, Pearce I, Priglinger S, Wenzel A, Barnes E, Gekkieva M, Pilz S, et al BRIGHTER study group: Individualized stabilization criteria-driven ranibizumab versus laser in branch retinal vein occlusion: Six-month results of BRIGHTER. Ophthalmology 123: 1332-1344, 2016

17. Brown DM, Ou WC, Wong TP, Kim RY, Croft DE and Wykoff CC; DAVE Study Group: Targeted retinal photocoagulation for diabetic macular edema with peripheral retinal nonperfusion: Three-year randomized DAVE trial. Ophthalmology 125: 683-690, 2018

18. Yan M, Huang Z, Lian HY, Song YP and Chen X: Conbercept for treatment of choroidal neovascularization secondary to pathologic myopia. Acta Ophthalmol 97: e813-e814, 2019.
19. Lang GE, Liakopoulos S, Vögeler J, Weiß C, Spital G, Gamulescu MA, Lohmann C and Wiedemann P: The RELATION study: Efficacy and safety of ranibizumab combined with laser photocoagulation treatment versus laser monotherapy in NPDR and PDR patients with diabetic macular oedema. Acta Ophthalmol 96: e377-e385, 2018.

20. Ishibashi T, Li X, Koh A, Lai TY, Lee FL, Lee WK, Ma Z, Ohji M, Tan N, Cha SB, et al; REVEAL Study Group: The REVEAL Study: Ranibizumab monotherapy or combined with laser versus laser monotherapy in Asian patients with diabetic macular edema. Ophthalmology 122: 1402-1415, 2015.

21. Januschowski K, Feltgen N, Pielen A, Spitzer B, Rehak M, Spital G, Dimopoulos S, Meyer CH and Szurman GB; Bevacizumab Study Group Venous Occlusion: Predictive factors for functional improvement following intravitreal bevacizumab injections after central retinal vein occlusion. Graefes Arch Clin Exp Ophthalmol 255: 457-462, 2017.

22. Theodossiadis PG, Grigoropoulos VG and Theodossiadis GP: The significance of the external limiting membrane in the recovery of photoreceptor layer after successful macular hole closure: A study by spectral domain optical coherence tomography. Ophthalmologica 225: 176-184, 2011.

23. Inoue M, Watanabe Y, Arakawa A, Sato S, Kobayashi S and Kadonosono K: Spectral-domain optical coherence tomography images of inner/outer segment junctions and macular hole surgery outcomes. Graefes Arch Clin Exp Ophthalmol 247: 325-330, 2009.

This work is licensed under a Creative Commons Attribution-NonCommercial-NoDerivatives 4.0 International (CC BY-NC-ND 4.0) License. 